\title{
Prediction Variance Capabilities of Third-Order Response Surface Designs for Cuboidal Regions
}

\author{
Brenda Mbouamba Yankam ${ }^{1,2, *}$, Abimibola Victoria Oladugba ${ }^{2}$ \\ ${ }^{1}$ Cameroon Baptist Convention Health Services (CBCHS), Littoral Region, Cameroon \\ ${ }^{2}$ Department of Statistics, University of Nigeria, Nsukka, Nigeria
}

Received June 30, 2021; Revised August 23, 2021; Accepted September 21, 2021

\section{Cite This Paper in the following Citation Styles}

(a): [1] Brenda Mbouamba Yankam, Abimibola Victoria Oladugba , "Prediction Variance Capabilities of Third-Order Response Surface Designs for Cuboidal Regions," Mathematics and Statistics, Vol. 9, No. 5, pp. 760 - 772, 2021. DOI: 10.13189/ms.2021.090516.

(b): Brenda Mbouamba Yankam, Abimibola Victoria Oladugba (2021). Prediction Variance Capabilities of Third-Order Response Surface Designs for Cuboidal Regions. Mathematics and Statistics, 9(5), 760 - 772. DOI: 10.13189/ms.2021.090516.

Copyright $\bigcirc 2021$ by authors, all rights reserved. Authors agree that this article remains permanently open access under the terms of the Creative Commons Attribution License 4.0 International License

\begin{abstract}
Experimenters often evaluate the steadiness and consistency of designs over the region of interest by means of the prediction variance capabilities using the variance dispersion graph and the fraction of design space graph. The variance dispersion graph and the fraction of design space graph effectively describe the prediction variance capabilities of a design in the region of interest. However, the prediction variance capabilities of third-order response surface designs have not been studied in the literature. In this paper, the prediction variance capabilities of two third-order response surface designs term augmented orthogonal uniform composite designs and orthogonal array composite designs in the cuboidal region for $3 \leq k \leq 7$ with $n_{0}=5$ center points are examined. The prediction variance capabilities are evaluated using the variance dispersion graph and the fraction of design space graph. Also, $D-, E-, G$-and $T$-optimality criteria are used in evaluating these designs in terms of single-value criterion. The results obtained show that the augmented orthogonal uniform composite designs have better prediction variance capabilities in the cuboidal region in the terms of the variance dispersion graphs for factors 3 and 4 . The augmented orthogonal uniform composite designs also have better prediction variance capabilities for $3 \leq k \leq 7$ compare to the orthogonal array composite designs in terms of the fraction of design space graph. The augmented orthogonal uniform composite designs are shown to be superior over the orthogonal array composite designs in terms of $D$-, $E$-,
\end{abstract}

$G$-and $T$-optimality criteria for single-value criterion. This shows that the performances of the prediction variance capabilities of third-order response surface designs can be clearly visualized by means of the variance dispersion graph and fraction of design space and should be consider over the single-value criteria even though the single value-criteria show some degree of design performance. The augmented orthogonal uniform composite design is should often be considered in experimentation over the orthogonal array composite design since the augmented orthogonal uniform composite design performance better.

Keywords Response Surface Designs, Prediction Variance, Third-order Designs, Optimality Criteria, Variance Dispersion Graph, Fraction of Design Space Plot

\section{Introduction}

The response surface methodology (RSM) incorporates statistically designed experiments to make inferences about model parameter. The RSM was first introduced by Box and Wilson [1] with the aims to improve, develop and optimize experimental processes [2]. In order to optimize these experimental processes, the response of interest and the inputs variables are examined by using the third-order response surface designs when the second-order response surface designs fail as a result of lack of fit caused by 
higher-order terms observed in the true mean response model $[3,4]$. The third-order response surface designs are designs that estimate effectively all the parameters of the third-order model [4]. They are often used in many scientific domains like engineering and computer sciences to optimize experimental processes and are frequently selected based on certain criteria of interest, such as the prediction variance capabilities and the optimality criteria $[5,6,7]$. The steadiness and consistency of the prediction variance capabilities over the region of interest is one of the most commonly used criteria.

Take, for instance, Park et al. [8] extensively studied the prediction variance capabilities of some second-order response surface designs in the cuboidal region by using the variance dispersion graph (VDG) and the fraction of design space (FDS) graph and show that Hoke designs and G-efficient designs should be considered when experimenting over a cuboidal region. Also, the face-centered cube or Hoke designs are recommended for a small number of variables but when the number of variables increases, the Hoke design is a better alternative to the face-centered cube; Wesley et al. [9] studied the exact calculation of integrated prediction variance for some response surface designs in the cuboidal and spherical regions and show that the central composite designs performed better than the Box-Behnken designs in the spherical region while the face centered cube and Hoke designs performed better in the cuboidal region; Borkowski [6] graphically assessed the prediction capabilities of some response surface designs; Zahran et al. [10] studied the prediction variance properties of some response surface designs using the fraction of design space (FDS) graph and show that the FDS graph show a better picture of the prediction variance in the cuboidal and spherical region than the single value criteria; Myers et al. [5] evaluated the prediction variance properties of second-order central composite design (CCD), Box-Behnken design (BBD), small composite designs (SCD), and hybrid designs in the spherical regions of interest and show that the CCD outperformed the other second-order response surface designs; Goldfarb et al. [11] studied the scaled prediction variance properties of mixture and mixture-process designs using the fraction of design space (FDS) plot in the cuboidal region and show that the FDS plot are easy to visualize than the VDG in the mixture and mixture-process designs; Sang - Hyun and Dac Heung [12] studied the prediction variance properties of some blocked response surface designs in the cuboidal region. However, the prediction variance capabilities of third-order response surface designs especially in the cuboidal region have not yet been studied in the literature.

This paper, therefore, aims at evaluating the prediction variance capabilities of two third-order response surface designs, augmented orthogonal uniform composite design denoted by AOUCD introduced by Yankam [13] and orthogonal array composite design $\left(\mathrm{OACD}_{4}\right)$ introduced by Zhang et al. [4]. The striking difference between the AOUCD and the $\mathrm{OACD}_{4}$ is the presence of the uniform design in the AOUCD that causes the AOUCD to be a good space-filling design since the design points are evenly distributed in the experimental region and also the fact that the AOUCD provides more flexible run sizes compared to the $\mathrm{OACD}_{4}$. However, these two designs estimate better the parameters of the third-order model and have the ability to perform multiple analysis with different parts of the data for cross-validation. The prediction variance capabilities of these third-order response surface designs for any region of interest, are evaluated graphically using the variance dispersion graph (VDG) and the fraction of design space (FDS) graph. These third-order response surface designs are also evaluated in terms of single-value criteria such as the $D$-, $E$-, $G$ - and $T$-optimality criteria to determine which third-order response surface design is superior or performs better to the other. Note that the single value criteria aid in selecting superior design although they lack the ability to project the true nature of the design in the experimental region.

The remainder of this paper is organized into 5 sections as follows: Section 2 gives a brief introduction of augmented orthogonal uniform composite designs (AOUCDs) and orthogonal array composite designs $\left(\mathrm{OACD}_{4}\right)$. Section 3 describes the prediction variance capabilities using the variance dispersion graph (VDG) and the fraction of design space (FDS) graph to evaluate the prediction variance of AOUCDs and $\mathrm{OACD}_{4}$. In Section 4, the $D$-, $E$-, $G$ - and $T$-optimality criteria used in this study is discussed. Section 5 provides the conclusion of this study.

\subsection{Third-order Model Development}

The model for the observed response in the design variables of the third-order model fitted onto the AOUCD is defined in equation (1) as;

$$
y=\beta_{0}+\sum_{i=1}^{k} \beta_{i} x_{i}+\sum_{i=1}^{k} \beta_{i i} x_{i}^{2}+\sum_{i=1}^{k-1} \sum_{j=i+1}^{k} \beta_{i j} x_{i} x_{j}+\sum_{i=1}^{k} \beta_{i i i} x_{i}^{3}+\varepsilon
$$


It can be express in matrix form as;

$$
y=X \beta+\varepsilon
$$

where $y=\left(y_{1}, \ldots, y_{n}\right)^{\prime}$ is the vector of the observed responses of interest, $\beta$ is the $p \times 1$, $p=1+3 k+3\left(\begin{array}{l}k \\ 2\end{array}\right)+\left(\begin{array}{l}k \\ 3\end{array}\right)$, vector of unknown parameter which appears in the chosen model, and $\varepsilon=\left(\varepsilon_{1}, \ldots, \varepsilon_{n}\right)^{\prime}$ is the vector of random error associated with $y$. The error, $\varepsilon$, is assumed to be independent, identically distributed random variables with mean 0 and variance $\sigma^{2}$. The matrix $X$ is the model matrix.

The prediction variance at a given point $x$ in the experimental region is defined in equation (3) as;

$$
\operatorname{var}[\hat{y}(x)]=\sigma^{2} x^{\prime}\left(X^{\prime} X\right)^{-1} x
$$

Where

$x=\left(1, x_{1}, x_{2}, \ldots, x_{k} ; x_{1}^{2}, x_{2}^{2}, \ldots, x_{k}^{2} ; x_{1} x_{2}, \ldots, x_{k-1} x_{k} ; x_{1}^{3}, x_{2}^{3}, \ldots, x_{k}^{3}\right)$

is the vector of design points in the design space. Scaling the prediction variance is often helps to make comparisons between designs of varying sizes and this is frequently used in evaluating the design's prediction capabilities. Hence, the scaled prediction variance (SPV) is obtained from equation (3) by multiplying it by, $n$ which is the design run size and dividing by the process variance, $\sigma^{2}$. The SPV used to compare designs is defined in equation (4) as:

$$
\frac{\operatorname{var}[\hat{y}(x)]}{\sigma^{2}}=n x^{\prime}\left(X^{\prime} X\right)^{-1} x
$$

The SPV is used as a mean to plot the variance dispersion graph (VDG) and Fraction of design space (FDS) graph. The VDG is comprised of three properties, i.e., the maximum SPV, average SPV and minimum SPV. The maximum SPV, average SPV and minimum SPV are defined in equations (5), (6) and (7) respectively as:

$$
\begin{gathered}
\text { Maximum SPV }=\max \frac{n \operatorname{var}[\hat{y}(x)]}{\sigma^{2}}=1+n\left(\lambda_{\max }\right) r^{2} \\
\text { Average SPV }=V^{r}=1+\frac{n r^{2}}{k} \sum_{i=1}^{k} \lambda_{\text {ave }}
\end{gathered}
$$

and

$$
\text { Minimum SPV }=\min \frac{n \operatorname{var}[\hat{y}(x)]}{\sigma^{2}}=1+n\left(\lambda_{\min }\right) r^{2}
$$

where $\lambda_{\text {max }}, \lambda_{\text {ave }}$ and $\lambda_{\text {min }}$ are the maximum, average and minimum eigenvalues of $\left(X^{\prime} X\right)^{-1}$ respectively.

In comparing designs of different sizes, the scaling in prediction variance is often useful in determining whether the additional runs in a larger design are of any importance in significantly reducing the variance of the predicted response [14-16].

\subsection{Graphical Techniques}

Two graphical techniques are used to evaluate the prediction variance capabilities of third-order augmented orthogonal uniform composite designs (AOUCDs) and orthogonal array composite designs $\left(\mathrm{OACD}_{4}\right)$. The first graphical technique is the variance dispersion graph (VDG) which is used to evaluate the prediction variance capabilities of response surface designs and it was first introduced by Giovannitti-Jensen and Myers [17]. The VDG is a three-dimensional graph used in evaluating the steadiness and consistency of the variance of a predicted response of designs and is composed of three properties, the maximum, average and minimum prediction variance on a hypercube given a cuboidal radius $r$. Schoonees et al. [18] stated that a design with a steady and consistent SPV is often desired over those that are not and this steadiness and consistency are often associated with a smooth and flat VDG curve. The second graphical technique is the fraction of design space (FDS) graph that is used to evaluate the prediction variance capabilities of response surface designs over the fraction of space and it was first introduced by Zahran et al. [9] to complement the VDG. The FDS graph is a two-dimensional graph with a single curve over a multi-dimensional region and this graph shows the fraction of the design space at a prediction variance value. The FDS graph shows the fraction of the design space at a given scaled prediction value, $g$, and as mention by Zahran et al. [9], the larger the fraction of design space at a given value, the better the design.

The VDG and FDS graphs each describe a different aspect of the scaled prediction variance (SPV) distribution for a design. The SPV help experimenters to see if adding runs to an experiment will result in an actual reduction in the predicted response's variance. The FDS graph differs from VDG in that the FDS gives equal weight to all locations in the design space compare to the VDG. These graphs provide a better overview of a design's prediction variance performance.

\section{Third-order Response Surface Designs}

The construction method of augmented orthogonal uniform composite designs (AOUCDs) and orthogonal array composite designs $\left(\mathrm{OACD}_{4}\right)$ are described in this section.

\subsection{Augmented Orthogonal Uniform Composite Designs (AOUCDs)}

A class of third-order response surface design called augmented orthogonal uniform composite design (AOUCD) was introduced by Yankam [13]. The AOUCD is made up of three parts: $n_{f}$ factorial portion with factor levels defined by $[-1,1]$; four-level uniform design (UD) as the axial portion with all $x_{i}=-\alpha,-\frac{\alpha}{a}, \frac{\alpha}{a}$, or $\alpha(\alpha>0 ; a>1 ; i=1,2, \ldots, k)$ 
denoted by $n_{\alpha}$ and $n_{0}$ center points with all $x_{i}=0$ resulting in $N=n_{f+} n_{\alpha}+n_{0}$.

An AOUCD is constructed by selecting a two-level factorial design and a four-level uniform design (UD). A two-level factorial design is constructed through the selection of either a $2^{k}$ full factorial if $k \leq 4$ or a regular $2^{k-l}$ fractional factorial design, which are both minimum aberration designs with the high resolution having the highest possible aliasing structure. Here, $k$ represents the number of factors and $l$ the design generator. The two-level factorial design was constructed by using Minitab version 16 statistical software.

Let $U D\left(N, S_{1}, \ldots, S_{k}\right)$ be a $k$ factor $U D$-type designs whereby the $S_{j}$ levels, $\left(1, \ldots, S_{j}\right)$, occur $N / S_{j}$ times in the $j^{\text {th }}$ column, $(j=1, \ldots, k)$, represented by $U D\left(N, S^{k}\right)$ when $S_{1}$
$=\ldots=S_{k}=S$, with $U D$ being the uniform design, $N$ the number of runs, $S$ the level of the factors and $k$ the number of factors. A uniform $U D$-type design is constructed under the mixture discrepancy criterion (MD) by using the following arguments init= "rand", crit= "MD", and maxiter $=100$ from the $\mathrm{R}$ package named UniDOE of Zhang et al. [19]. The axial portion i.e., $\alpha$ is chosen at the cuboidal region such that $\alpha=1$. Hence, the cuboidal AOUCD is provided by all axial design points on a hypercube. Take for instance, the 37-run AOUCD factor 5 presented in Table 1 . This is a simple combination of a minimum aberration $2_{V}^{5-1}$ design with a mixture discrepancy five-column uniform design, $U D\left(16,4^{5}\right)$ for factor 5 with $n_{0}=5$ center points.

Table 1. A general structure of 37-runs AOUCD for factor 5 with $n_{0}=5$ center point

\begin{tabular}{|c|c|c|c|c|c|c|}
\hline & \multirow{3}{*}{ Runs } & \multicolumn{5}{|c|}{ Factors } \\
\hline & & \multicolumn{5}{|c|}{5 factors AOUCD with 37-runs } \\
\hline & & A & $\mathbf{B}$ & C & D & $\mathbf{E}$ \\
\hline \multirow{16}{*}{ Factorial portion $\left(n_{f}\right)$} & 1 & 1 & -1 & -1 & -1 & -1 \\
\hline & 2 & -1 & 1 & -1 & -1 & -1 \\
\hline & 3 & -1 & -1 & 1 & -1 & -1 \\
\hline & 4 & -1 & -1 & -1 & 1 & -1 \\
\hline & 5 & -1 & -1 & -1 & -1 & 1 \\
\hline & 6 & 1 & 1 & 1 & -1 & -1 \\
\hline & 7 & 1 & 1 & -1 & 1 & -1 \\
\hline & 8 & 1 & 1 & -1 & -1 & 1 \\
\hline & 9 & 1 & -1 & 1 & 1 & -1 \\
\hline & 10 & 1 & -1 & 1 & -1 & 1 \\
\hline & 11 & 1 & -1 & -1 & 1 & 1 \\
\hline & 12 & -1 & 1 & 1 & 1 & -1 \\
\hline & 13 & -1 & 1 & 1 & -1 & 1 \\
\hline & 14 & -1 & 1 & -1 & 1 & 1 \\
\hline & 15 & -1 & -1 & 1 & 1 & 1 \\
\hline & 16 & 1 & 1 & 1 & 1 & 1 \\
\hline \multirow{16}{*}{ Axial portion $\left(n_{\alpha}\right)$} & 17 & $1 / 3$ & -1 & 1 & 1 & $-1 / 3$ \\
\hline & 18 & $-1 / 3$ & $-1 / 3$ & -1 & -1 & $1 / 3$ \\
\hline & 19 & -1 & -1 & $-1 / 3$ & $1 / 3$ & $-1 / 3$ \\
\hline & 20 & 1 & 1 & -1 & $-1 / 3$ & 1 \\
\hline & 21 & 1 & 1 & $1 / 3$ & $1 / 3$ & -1 \\
\hline & 22 & $-1 / 3$ & 1 & $-1 / 3$ & 1 & 1 \\
\hline & 23 & -1 & -1 & $1 / 3$ & $-1 / 3$ & $-1 / 3$ \\
\hline & 24 & $1 / 3$ & $-1 / 3$ & -1 & $1 / 3$ & 1 \\
\hline & 25 & $-1 / 3$ & $1 / 3$ & $1 / 3$ & -1 & -1 \\
\hline & 26 & 1 & -1 & 1 & -1 & $1 / 3$ \\
\hline & 27 & $-1 / 3$ & $-1 / 3$ & 1 & 1 & $-1 / 3$ \\
\hline & 28 & 1 & $-1 / 3$ & $-1 / 3$ & $-1 / 3$ & -1 \\
\hline & 29 & -1 & $1 / 3$ & 1 & -1 & $1 / 3$ \\
\hline & 30 & $1 / 3$ & 1 & $-1 / 3$ & $-1 / 3$ & -1 \\
\hline & 31 & -1 & $1 / 3$ & -1 & 1 & $1 / 3$ \\
\hline & 32 & $1 / 3$ & $1 / 3$ & $1 / 3$ & $1 / 3$ & 1 \\
\hline \multirow{5}{*}{ Center runs replicate $\left(n_{0}\right)$} & 33 & 0 & 0 & 0 & 0 & 0 \\
\hline & 34 & 0 & 0 & 0 & 0 & 0 \\
\hline & 35 & 0 & 0 & 0 & 0 & 0 \\
\hline & 36 & 0 & 0 & 0 & 0 & 0 \\
\hline & 37 & 0 & 0 & 0 & 0 & 0 \\
\hline
\end{tabular}


Table 2. A general structure of 37-runs $\mathrm{OACD}_{4}$ for factor 5 with $n_{0}=5$ center point.

\begin{tabular}{|c|c|c|c|c|c|c|}
\hline & \multirow{3}{*}{ Runs } & \multicolumn{5}{|c|}{ Factors } \\
\hline & & \multicolumn{5}{|c|}{5 factors $\mathrm{OACD}_{4}$ with 37-runs } \\
\hline & & A & $\mathbf{B}$ & C & $\mathbf{D}$ & $\mathbf{E}$ \\
\hline \multirow{16}{*}{ Factorial portion $\left(n_{f}\right)$} & 1 & 1 & -1 & -1 & -1 & -1 \\
\hline & 2 & -1 & 1 & -1 & -1 & -1 \\
\hline & 3 & -1 & -1 & 1 & -1 & -1 \\
\hline & 4 & -1 & -1 & -1 & 1 & -1 \\
\hline & 5 & -1 & -1 & -1 & -1 & 1 \\
\hline & 6 & 1 & 1 & 1 & -1 & -1 \\
\hline & 7 & 1 & 1 & -1 & 1 & -1 \\
\hline & 8 & 1 & 1 & -1 & -1 & 1 \\
\hline & 9 & 1 & -1 & 1 & 1 & -1 \\
\hline & 10 & 1 & -1 & 1 & -1 & 1 \\
\hline & 11 & 1 & -1 & -1 & 1 & 1 \\
\hline & 12 & -1 & 1 & 1 & 1 & -1 \\
\hline & 13 & -1 & 1 & 1 & -1 & 1 \\
\hline & 14 & -1 & 1 & -1 & 1 & 1 \\
\hline & 15 & -1 & -1 & 1 & 1 & 1 \\
\hline & 16 & 1 & 1 & 1 & 1 & 1 \\
\hline \multirow{16}{*}{ Axial portion $\left(n_{\alpha}\right)$} & 17 & 1 & $-1 / 3$ & -1 & 1 & -1 \\
\hline & 18 & $-1 / 3$ & $-1 / 3$ & 1 & $-1 / 3$ & -1 \\
\hline & 19 & 1 & 1 & 1 & $1 / 3$ & $-1 / 3$ \\
\hline & 20 & -1 & $-1 / 3$ & $1 / 3$ & $1 / 3$ & $1 / 3$ \\
\hline & 21 & 1 & $-1 / 3$ & 1 & -1 & $1 / 3$ \\
\hline & 22 & $-1 / 3$ & $1 / 3$ & -1 & $1 / 3$ & $1 / 3$ \\
\hline & 23 & -1 & 1 & $1 / 3$ & -1 & $-1 / 3$ \\
\hline & 24 & $1 / 3$ & $1 / 3$ & $1 / 3$ & 1 & -1 \\
\hline & 25 & $1 / 3$ & -1 & $-1 / 3$ & $1 / 3$ & $-1 / 3$ \\
\hline & 26 & -1 & $-1 / 3$ & $-1 / 3$ & $-1 / 3$ & -1 \\
\hline & 27 & $1 / 3$ & -1 & $1 / 3$ & $-1 / 3$ & 1 \\
\hline & 28 & $-1 / 3$ & -1 & -1 & -1 & $-1 / 3$ \\
\hline & 29 & $-1 / 3$ & -1 & 1 & 1 & 1 \\
\hline & 30 & -1 & 1 & $-1 / 3$ & 1 & 1 \\
\hline & 31 & 1 & 1 & -1 & $-1 / 3$ & 1 \\
\hline & 32 & $1 / 3$ & $1 / 3$ & $-1 / 3$ & -1 & $1 / 3$ \\
\hline \multirow{5}{*}{ Center runs replicate $\left(n_{0}\right)$} & 33 & 0 & 0 & 0 & 0 & 0 \\
\hline & 34 & 0 & 0 & 0 & 0 & 0 \\
\hline & 35 & 0 & 0 & 0 & 0 & 0 \\
\hline & 36 & 0 & 0 & 0 & 0 & 0 \\
\hline & 37 & 0 & 0 & 0 & 0 & 0 \\
\hline
\end{tabular}

\subsection{Orthogonal Array Composite Designs $\left(\mathrm{OACD}_{4}\right)$}

The orthogonal array composite design $\left(\mathrm{OACD}_{4}\right)$ is a class of third-order response surface design that was introduced by Zhang et al. [4] and it is made up of three parts: a two-level orthogonal array (OA) as the factorial portion with factor levels given by $[-1,1]$ denoted by $n_{f}$; four-level orthogonal array (OA) as the axial portion with levels given

$x_{i}=-\alpha,-\frac{\alpha}{a}, \frac{\alpha}{a}$, or $\alpha(\alpha>0 ; a>1 ; i=1,2, \ldots, k)$

denoted by $n_{\alpha}$ and $n_{0}$ center points with $x_{i}=0$ resulting in $n_{f}+n_{\alpha}+n_{0}$. The four-level portion $n_{\alpha}$ of $\mathrm{OACD}_{4}$ was constructed by taking the first $k$ columns of "oa.16.5.4.2.txt" and "oa.32.9.4.2.txt" from Sloane's website http://neilsloane.com/oadir/. An example of the $\mathrm{OACD}_{4}$ for factor 5 with 5 center points is presented in Table 2.

\section{Design Evaluation}

The prediction variance capabilities of the AOUCDs and $\mathrm{OACD}_{4}$ for the third-order model are evaluated using the variance dispersion graph (VDG) and the fraction of design space (FDS) graph.

The VDGs of the AOUCDs and $\mathrm{OACD}_{4}$ when the region is cuboidal are plotted and presented in Figures 1 to 5 for $3 \leq k \leq 7$ with $n_{0}=5$ while the FDS graphs for AOUCDs and $\mathrm{OACD}_{4}$ are plotted and presented in Figures 6 to 10 .

From the graphs presented in Figures 1 to 5, we observed that the AOUCDs are superior to the $\mathrm{OACD}_{4}$ for factor 3 and 4 since the AOUCDs has a steadier and more consistent maximum, average and minimum SPV compared to those of the $\mathrm{OACD}_{4}$. However, the AOUCDs are not superior or do not perform well for factor 5, 6 and 7 as compared to the $\mathrm{OACD}_{4}$. This implies that the $\mathrm{OACD}_{4}$ has a steadier and more consistent maximum, average and 
minimum SPV compared to the AOUCDs.

Figures 6 to 10 show that the AOUCDs performed better compared to the $\mathrm{OACD}_{4}$ for $3 \leq k \leq 7$ with $n_{0}=5$. This implies that AOUCDs has a larger fraction of design space at a given SPV than the $\mathrm{OACD}_{4}$ meaning the AOUCDs are more superior over the $\mathrm{OACD}_{4}$ and this superiority was observed for $3 \leq k \leq 7$.

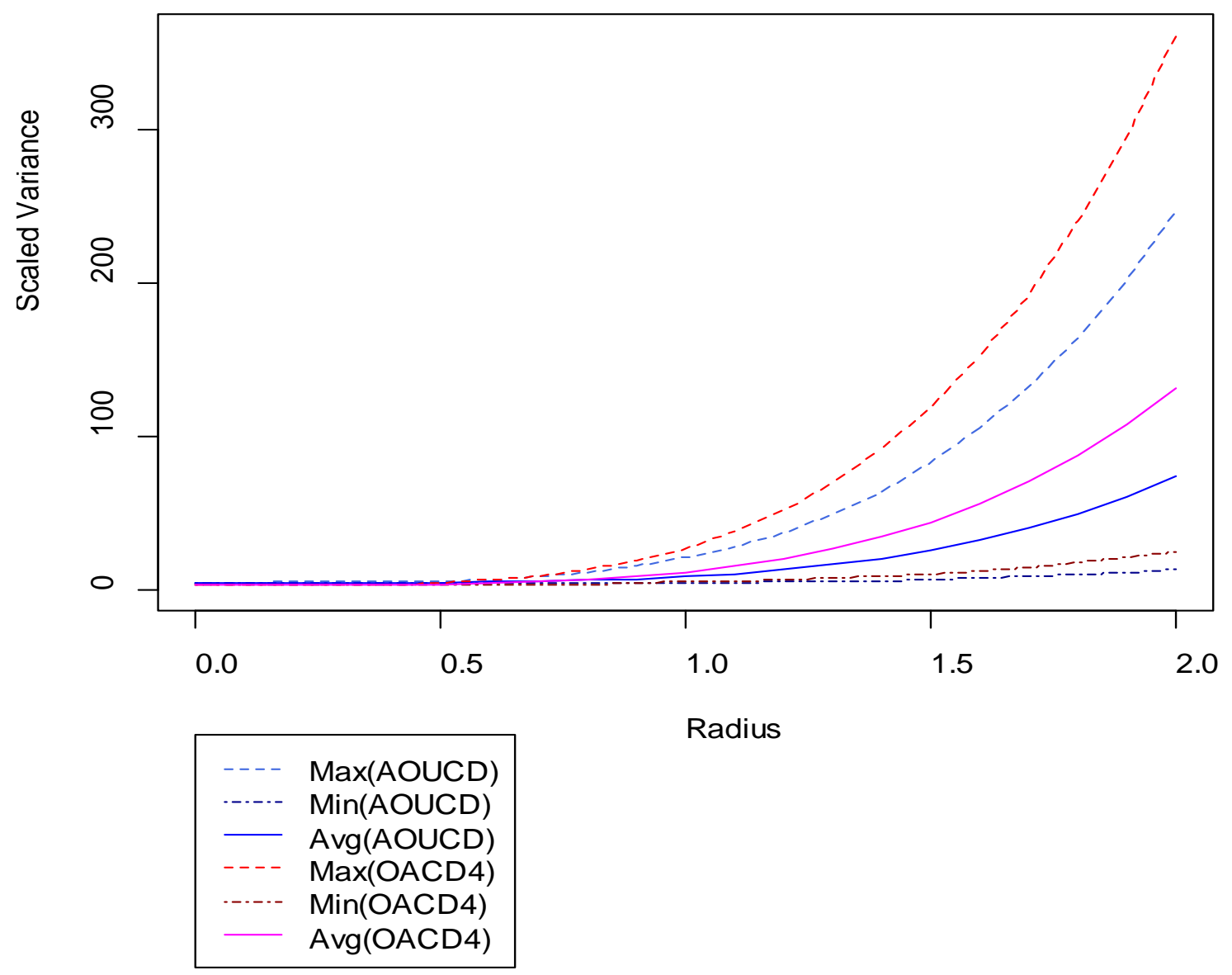

Figure 1. VDG for AOUCD and $\mathrm{OUCD}_{4}$ for factor 3 


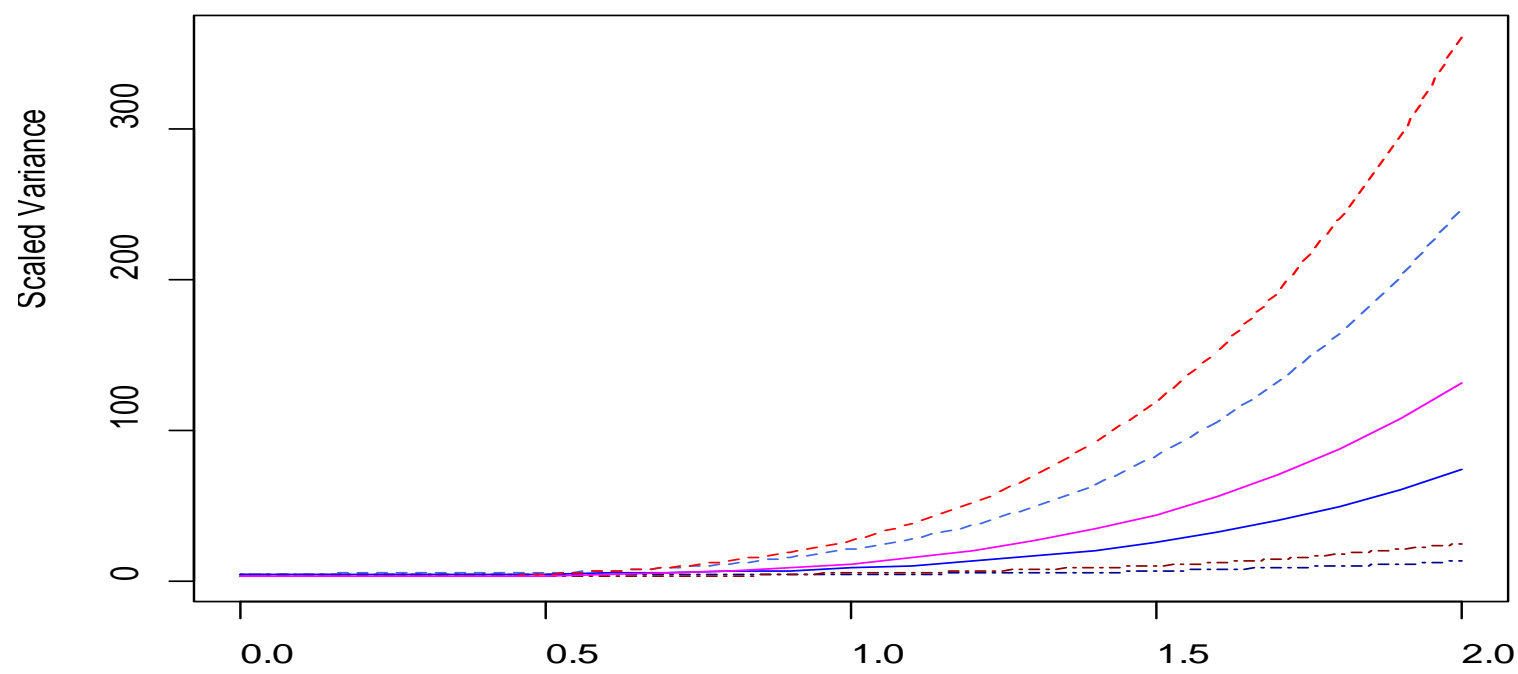

Radius

$$
\begin{aligned}
& \text {-- - } \operatorname{Max}(A O U C D) \\
& \operatorname{Min}(A O U C D) \\
& \text { Avg(AOUCD) } \\
& \operatorname{Max}(\mathrm{OACD} 4) \\
& \text { Min(OACD4) } \\
& \text { Avg(OACD4) }
\end{aligned}
$$

Figure 2. VDG for AOUCD and $\mathrm{OUCD}_{4}$ for factor 4

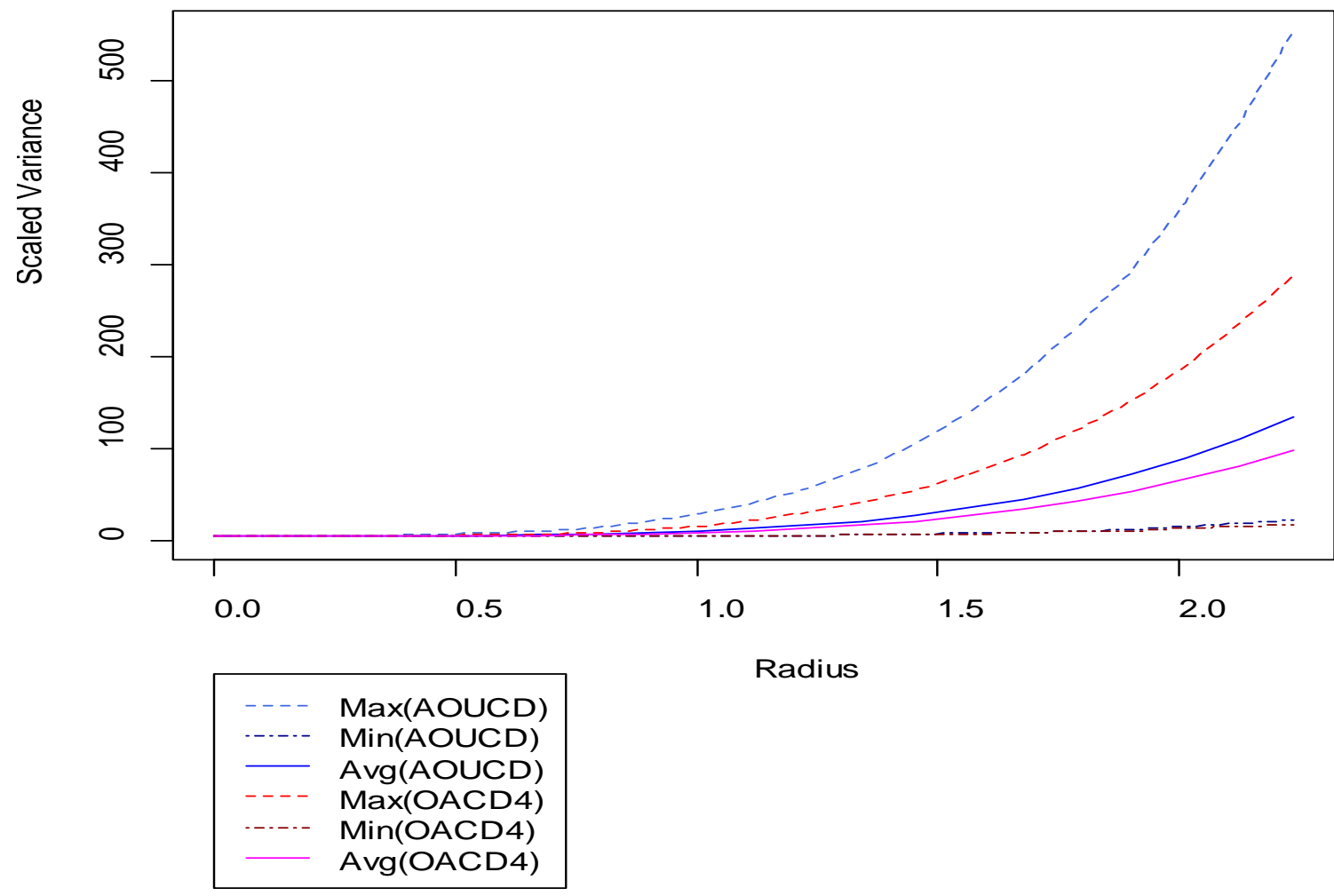

Figure 3. VDG for AOUCD and $\mathrm{OUCD}_{4}$ for factor 5 


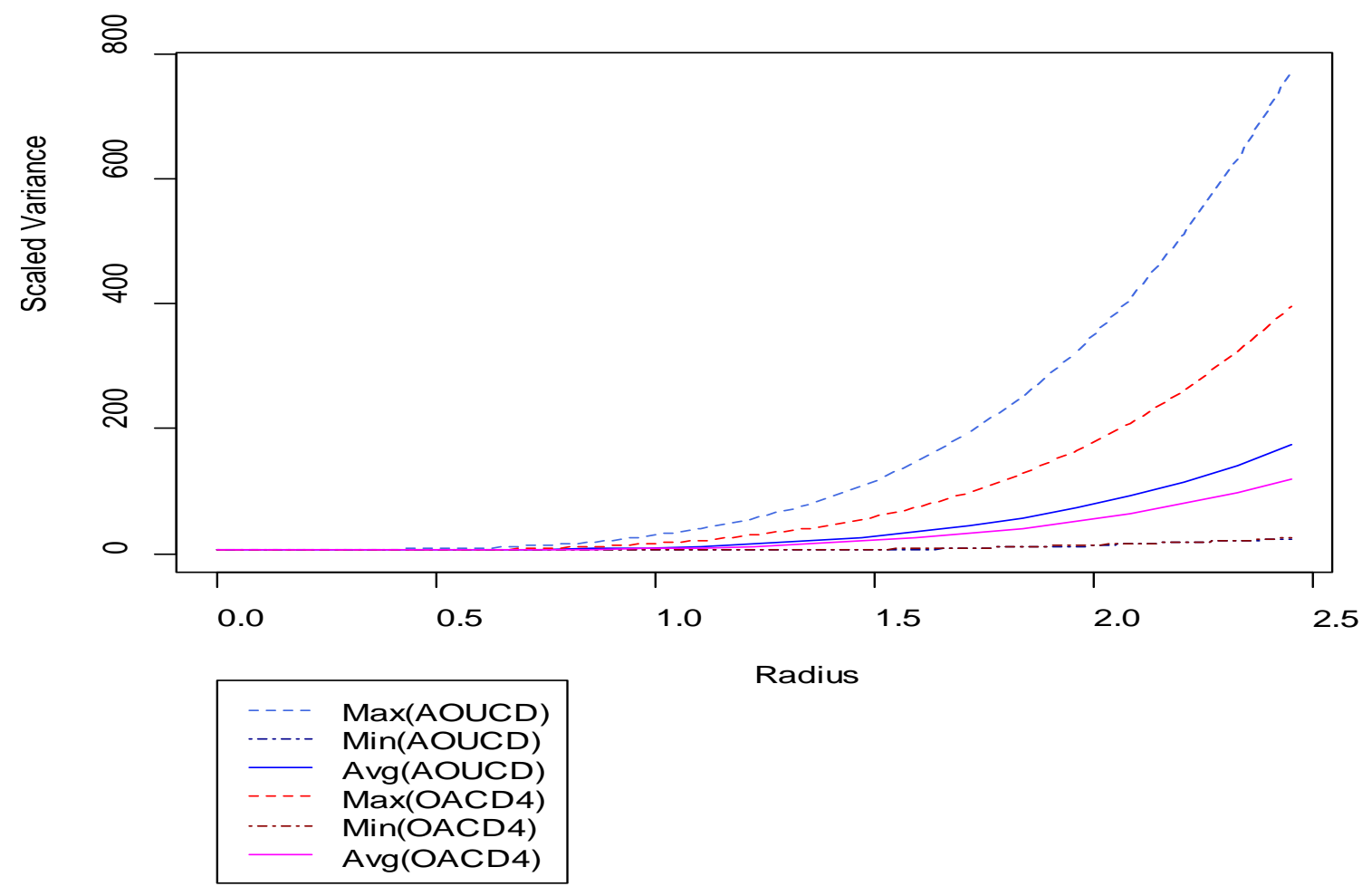

Figure 4. VDG for AOUCD and $\mathrm{OUCD}_{4}$ for factor 6

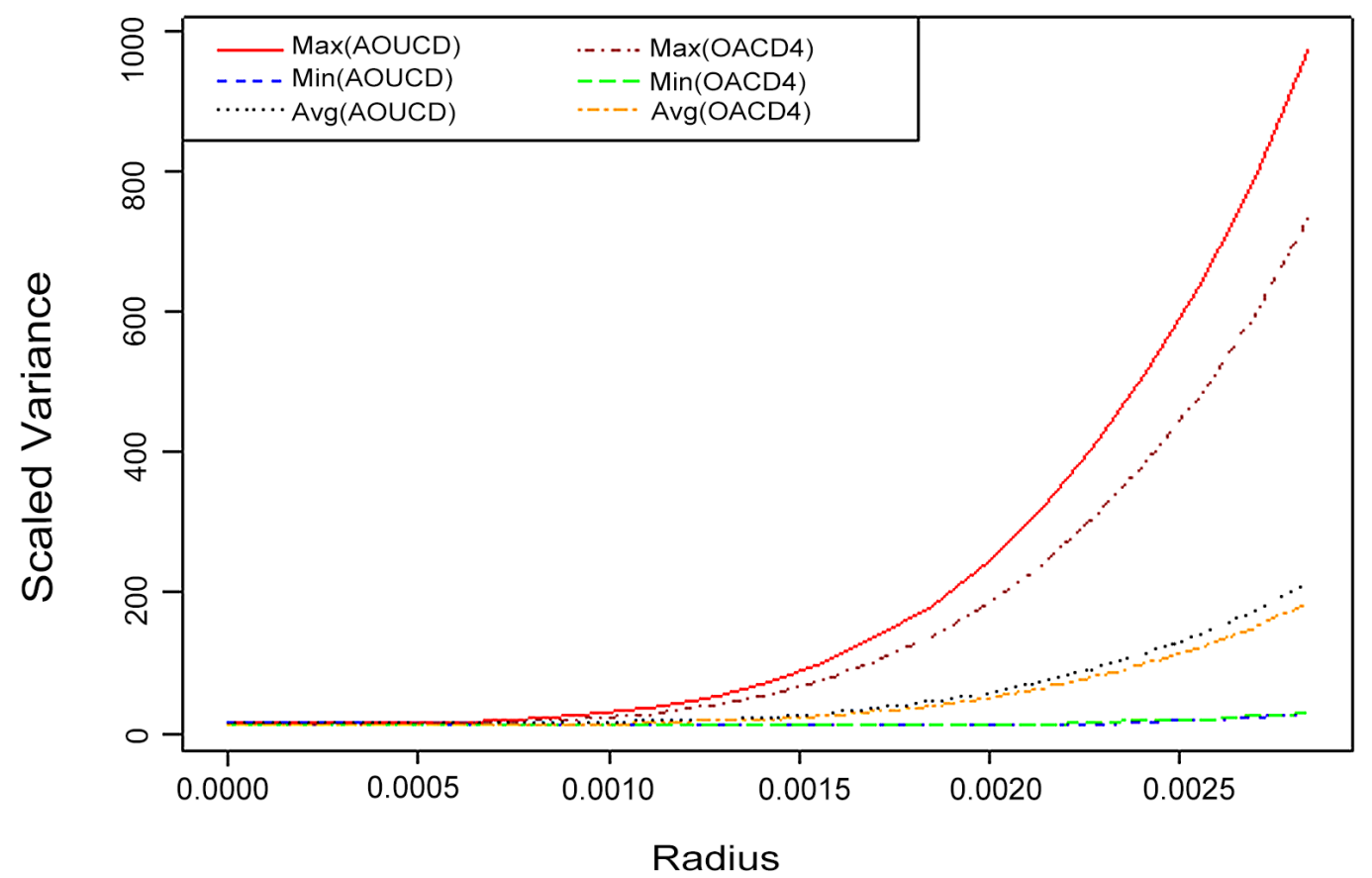

Figure 5. VDG for AOUCD and $\mathrm{OUCD}_{4}$ for factor 7 


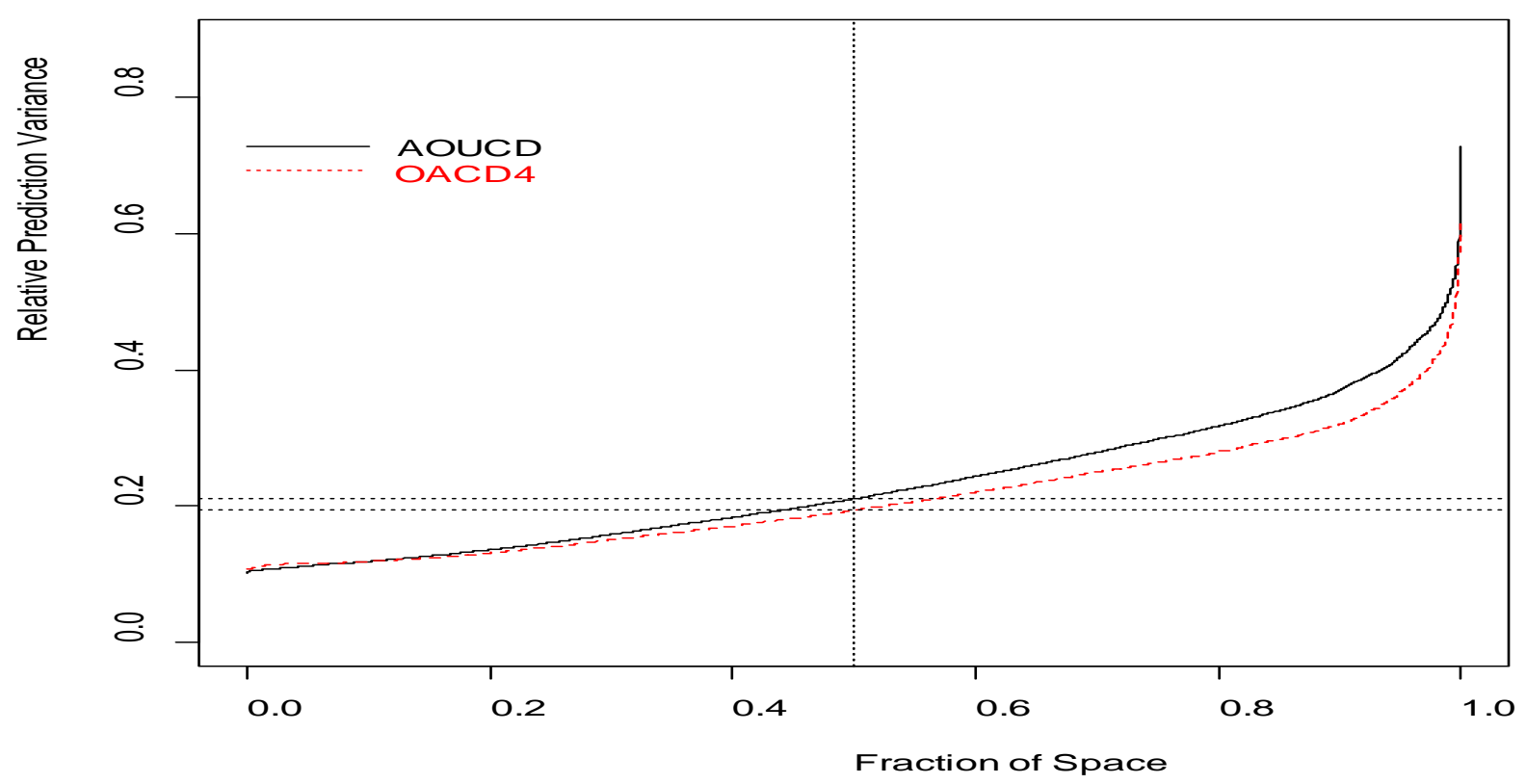

Figure 6. FDS for AOUCD and $\mathrm{OUCD}_{4}$ for factor 3

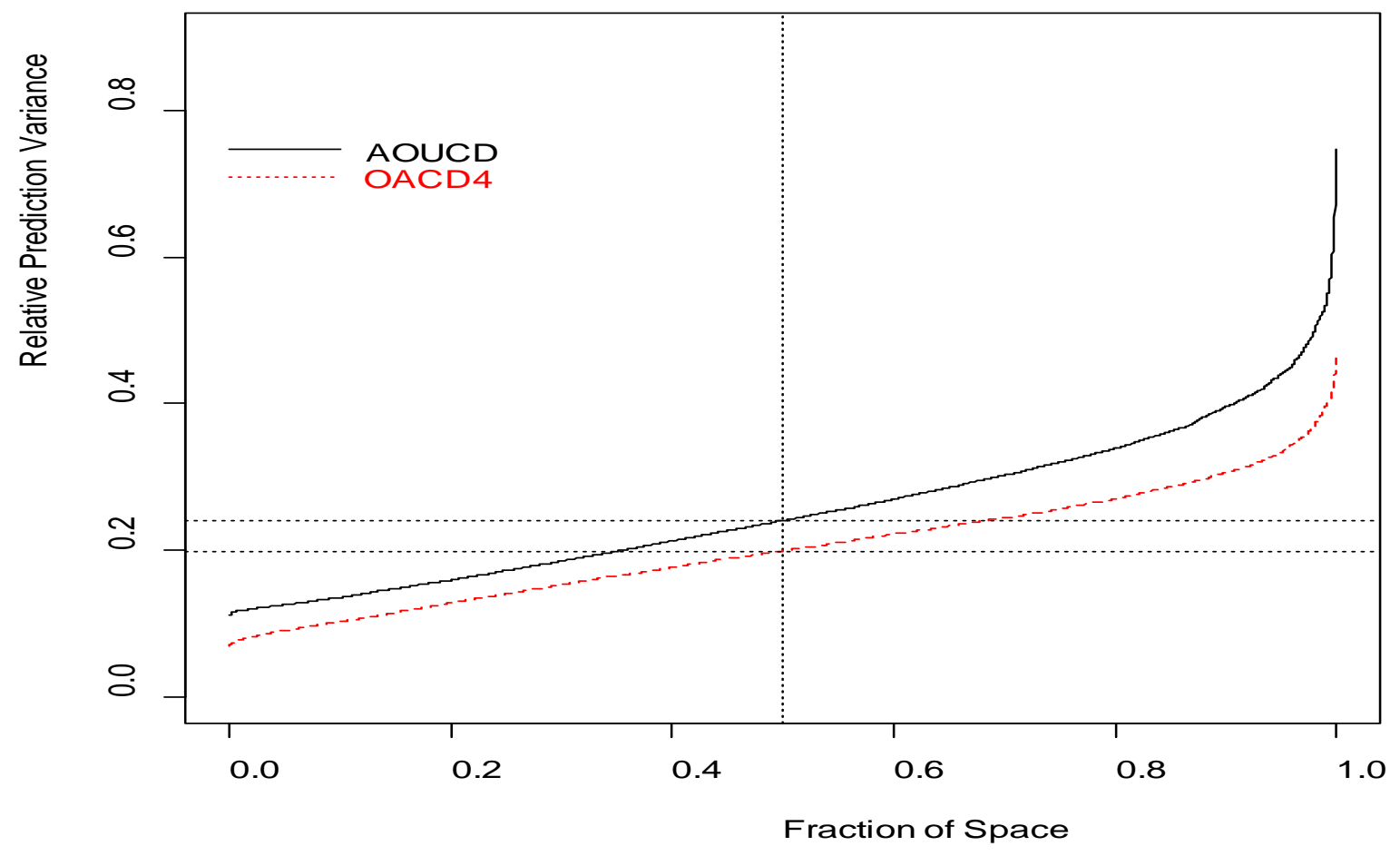

Figure 7. FDS for AOUCD and $\mathrm{OUCD}_{4}$ for factor 4 


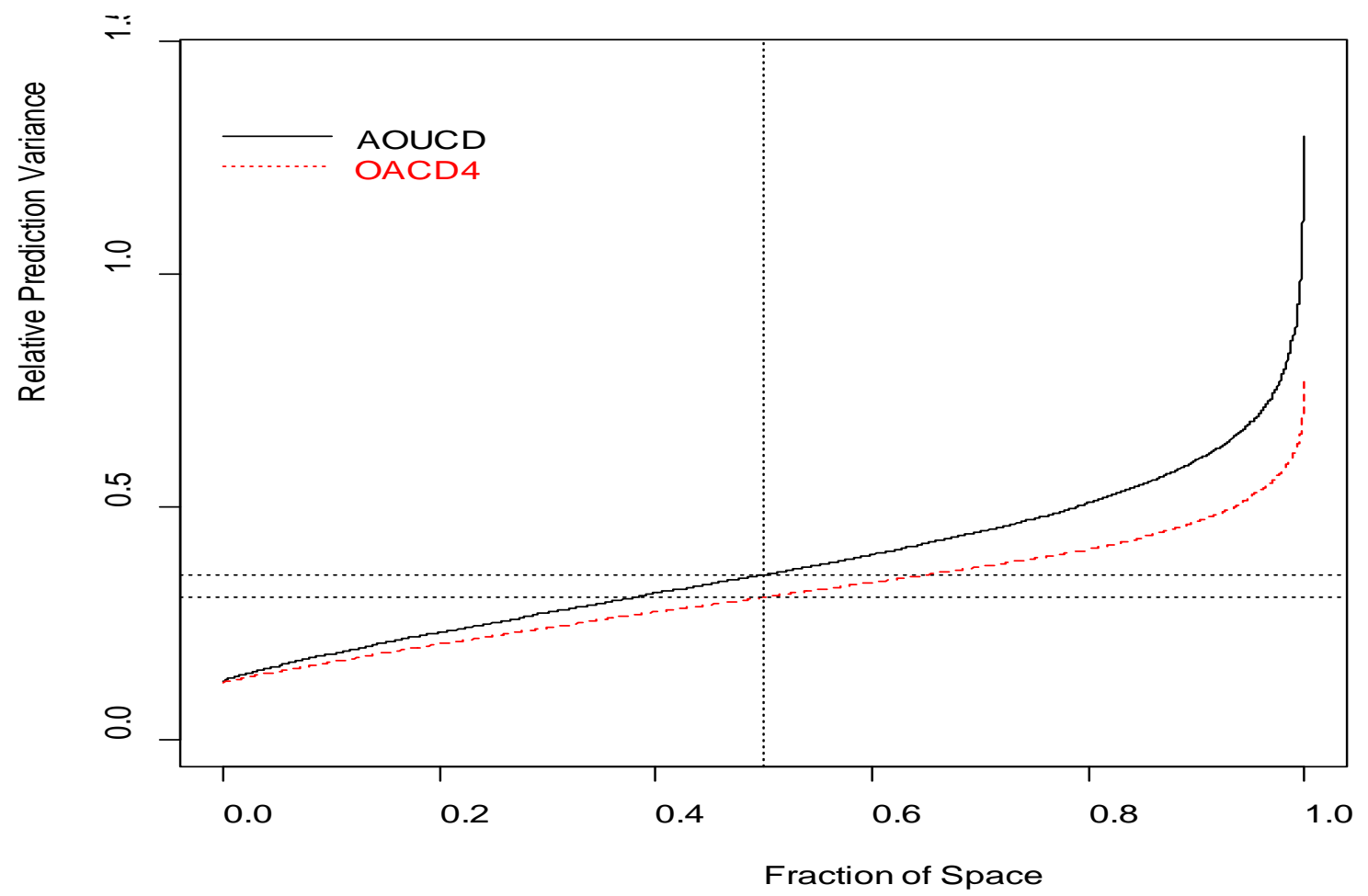

Figure 8. FDS for AOUCD and $\mathrm{OUCD}_{4}$ for factor 5

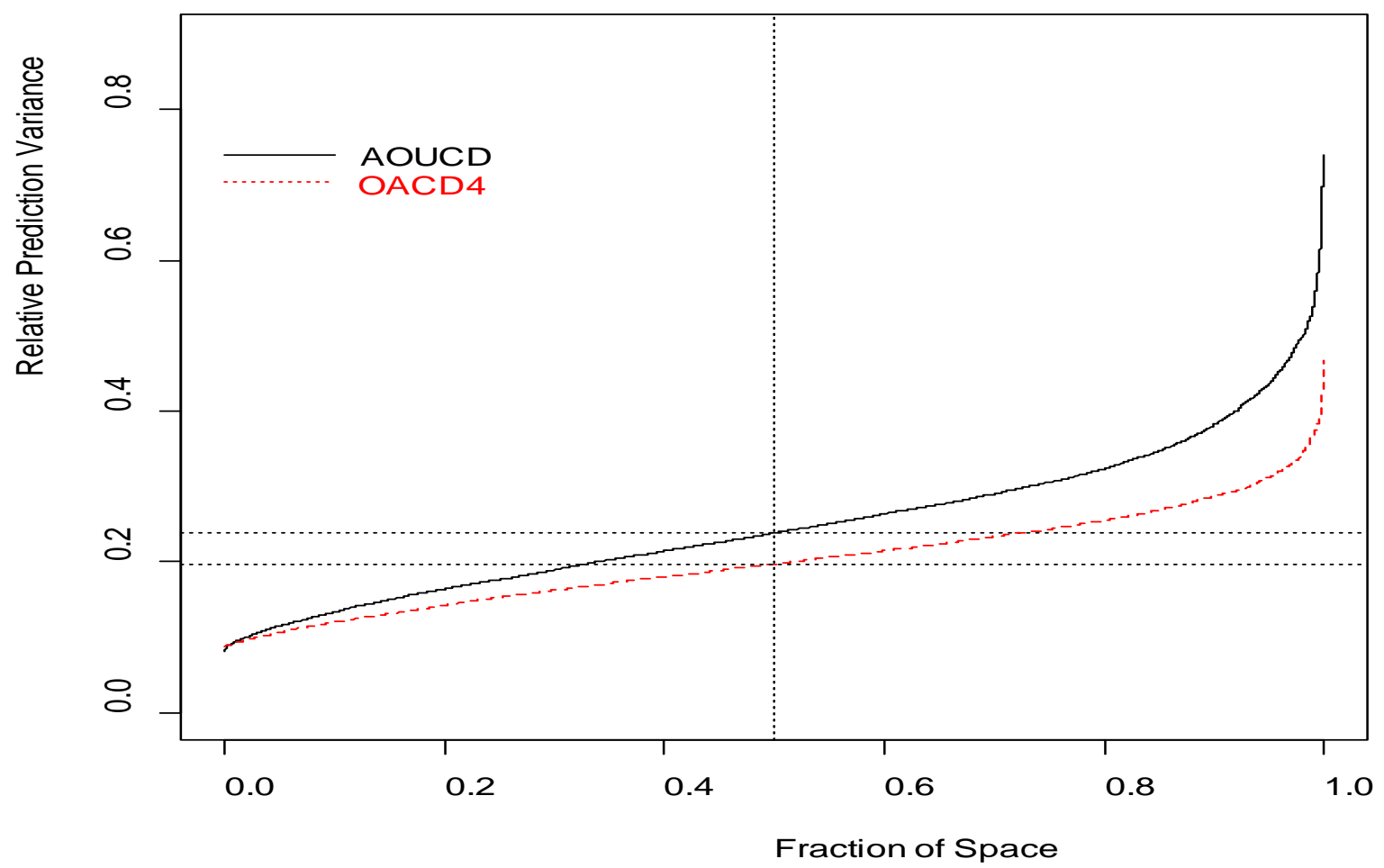

Figure 9. FDS for AOUCD and $\mathrm{OUCD}_{4}$ for factor 6 


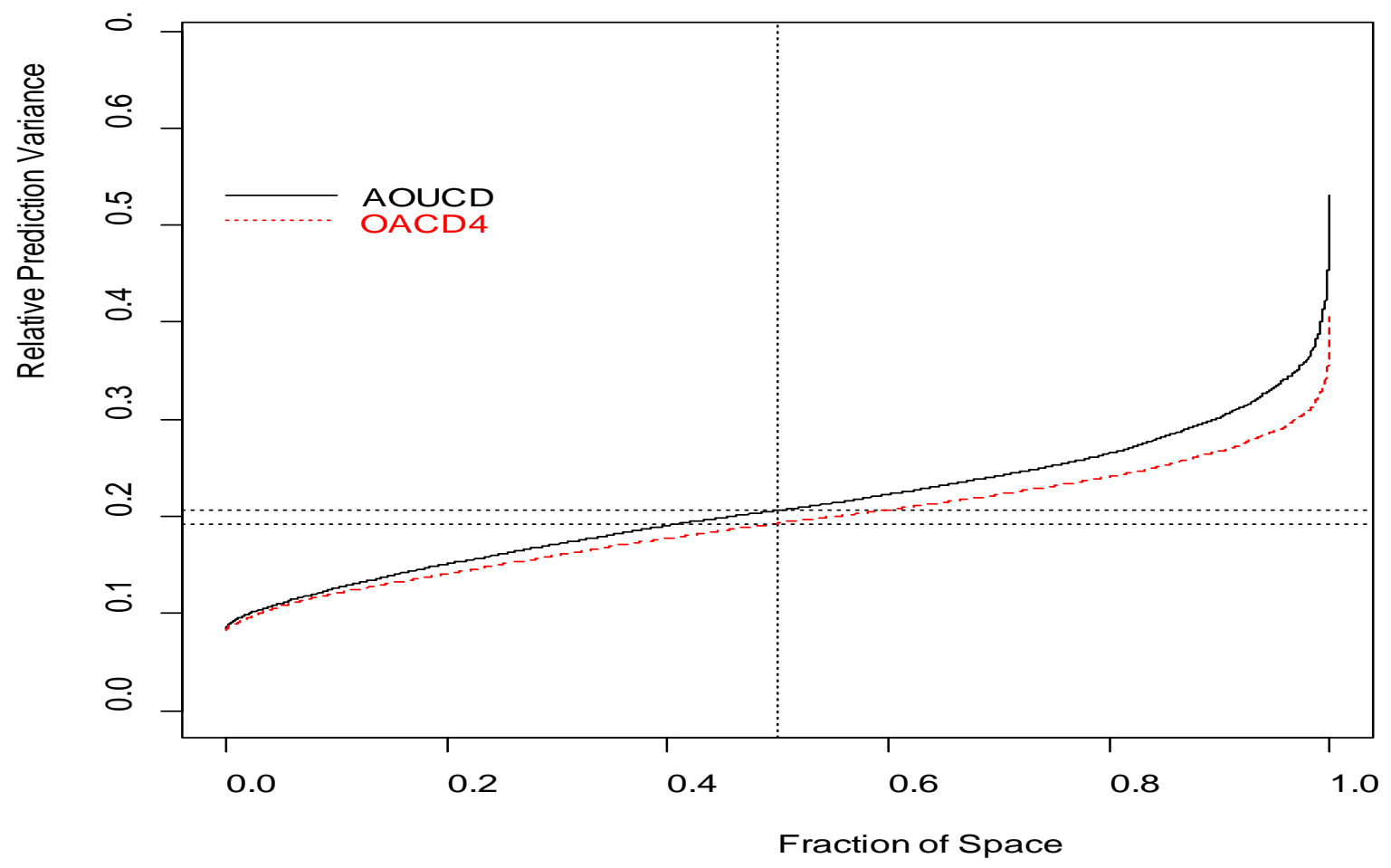

Figure 10. FDS for AOUCD and $\mathrm{OUCD}_{4}$ for factor 7

Table 3. Designs examined under the $D$-, E-, $G$ - and $T$-optimality criteria in the cuboidal region for $\alpha=1$ and $n_{0}=5$

\begin{tabular}{ccccccccc}
\hline Design & $\boldsymbol{k}$ & $\boldsymbol{p}$ & $\boldsymbol{n}_{\boldsymbol{f}}$ & $\boldsymbol{N}$ & $\boldsymbol{D}$-opt & $\boldsymbol{E}$-opt & $\boldsymbol{G}$-opt & $\boldsymbol{T}$-opt \\
\hline AOUCD & 3 & 13 & 8 & 29 & 0.1665 & 0.0029 & 0.1206 & 0.5740 \\
OACD $_{4}$ & 3 & 13 & 8 & 29 & 0.1960 & 0.0112 & 0.2057 & 0.5698 \\
\hline AOUCD & 4 & 19 & 16 & 37 & 0.1797 & 0.0017 & 0.0771 & 0.6456 \\
OACD $_{4}$ & 4 & 19 & 16 & 37 & 0.1852 & 0.0087 & 0.0526 & 0.5490 \\
\hline AOUCD & 5 & 26 & 16 & 37 & 0.1930 & 0.0015 & 0.0468 & 0.6314 \\
OACD $_{4}$ & 5 & 26 & 16 & 37 & 0.2187 & 0.0071 & 0.0901 & 0.6355 \\
\hline AOUCD & 6 & 34 & 32 & 96 & 0.2261 & 0.0027 & 0.0440 & 0.6729 \\
OACD $_{4}$ & 6 & 34 & 32 & 96 & 0.2517 & 0.0086 & 0.0857 & 0.6706 \\
\hline AOUCD & 7 & 43 & 64 & 101 & 0.2440 & 0.0014 & 0.0441 & 0.7696 \\
OACD $_{4}$ & 7 & 43 & 64 & 101 & 0.2688 & 0.0060 & 0.0583 & 0.7705 \\
\hline
\end{tabular}

\section{Single-value Criteria}

This section describes the $D$-, $E$-, $G$ - and $T$-optimality criteria used to evaluate the AOUCDs and $\mathrm{OACD}_{4}$ in terms of single-value criteria and the results of these single value criteria obtained for the AOUCDs and $\mathrm{OACD}_{4}$ are presented in Table 3. Borkowski [6] stated that in comparing response surface designs, designs with smaller single-value criteria are often desired as compared to those that are not.

\subsection{D-optimality Criterion}

The $D$-efficiency is the most commonly used criterion to evaluate response surface designs and this criterion either minimize $\left|\left(X^{\prime} X\right)^{-1}\right|$ or maximizes the determinant of the information matrix of a design and it is defined in equation (8) as;

$$
D_{e f f}(d)=\frac{1}{n}\left(\left|X^{\prime} X\right|\right)^{1 / p}
$$

where $X^{\prime} X$ is the information matrix, $\left|X^{\prime} X\right|$ the determinant of the information matrix and $p$ the number parameters given as $p=1+3 k+3\left(\begin{array}{l}k \\ 2\end{array}\right)+\left(\begin{array}{l}k \\ 3\end{array}\right)$.

\subsection{E-optimality Criterion}

$E$-efficiency is a criterion that maximizes the minimum eigenvalue of the information matrix of a design with the aim to minimize the maximum variance of all possible normalized linear combinations of parameter estimates. The E-efficiency of a design for the third-order model (1) with $k$-factor and $n$-runs is defined in equation (9) as;

$$
E_{\text {eff }}(d)=\min \text { eigen }\left[\left(X^{\prime} X\right) / n\right]
$$


where $X^{\prime} X$ is the information matrix and $n$ the number of experimental runs.

\subsection{G-optimality Criterion}

The $G$-optimality criterion is a prediction criterion. This criterion minimizes the maximum prediction variance over the experimental space $[20,21]$ and is defined in equation (10) as

$$
G_{\text {opt }}(d)=\frac{p}{v(\underline{x})_{\max }}
$$

where

$v(x)_{\max }=\max \left(n \cdot \operatorname{var} \frac{[\hat{y}(x)]}{\sigma^{2}}\right)=\left(n \cdot x^{\prime}\left(X^{\prime} X\right)^{-1} x^{\prime}\right)$ is the scale prediction variance, with $x$ being the location of the predicted response in the design space as well as the nature of the model, $X^{\prime} X$ is the information matrix and $p$ the number parameters given as; $p=1+3 k+3\left(\begin{array}{l}k \\ 2\end{array}\right)+\left(\begin{array}{l}k \\ 3\end{array}\right)$.

\subsection{T-optimality Criterion}

$T$-efficiency is a criterion designed to reflect the average diagonal elements of the information matrix. This criterion maximizes the trace of the information matrix for measuring the efficiency of design estimates and is defined in equation (11) as;

$$
T_{e f f}(d)=\frac{1}{n p} \operatorname{tr}\left(X^{\prime} X\right)
$$

where $n$ is the number of design points and $t r$ is the trace of a design.

From the results obtained for the $D-, E-, G$ - and $T$-optimality criteria of cuboidal AOUCDs and $\mathrm{OACD}_{4}$ for $3 \leq k \leq 7$ with $n_{0}=5$ in Table 2, it was observed that the AOUCDs performed better with smaller $D$-, $E$-, $G$ - and $T$-optimality criteria for most cases than the $\mathrm{OACD}_{4}$ which show that the AOUCDs are more desired over the $\mathrm{OACD}_{4}$. This implies that the AOUCDs are more superior over the $\mathrm{OACD}_{4}$ and that the impact of the uniform design in the AOUCD caused it to perform better as the design points are evenly distributed in the experimental region.

\section{Conclusions}

This paper evaluated the prediction variance capabilities of third-order augmented orthogonal uniform composite design denoted by AOUCD and orthogonal array composite designs denoted by $\mathrm{OACD}_{4}$ using the VDG and FDS in the cuboidal region, $\alpha=1$. The single-value criteria such as the $D$-, $E$-, $G$ - and $T$-optimality criteria are also used to evaluate the AOUCDs and $\mathrm{OACD}_{4}$. The AOUCDs were shown to perform better over the $\mathrm{OACD}_{4}$ since the AOUCDs had smaller $D$-, $E$-, $G$ - and $T$-optimality value compared to the $\mathrm{OACD}_{4}$, which is in line with what Borkowski [6] stated that in comparing response surface designs by optimality criteria, design with the smaller optimality value is most desired. This implies that the AOUCDs are better alternatives to $\mathrm{OACD}_{4}$ since it has better prediction variance capabilities and better optimality value in the cuboidal region than the $\mathrm{OACD}_{4}$. Although the single-value criteria are used in evaluating response surface designs, they sometimes fail to convey the true nature of the response surface design for estimating the response model in terms of prediction variance capabilities over a region of interest. As an alternative, the VDG and FDS graph are used to evaluate the prediction variance capabilities of the AOUCDs and $\mathrm{OACD}_{4}$ since they provide a clearer picture of the performances of the prediction variance capabilities. From the obtained results, the AOUCDs were shown to have better prediction variance capabilities in the experimental region over the $\mathrm{OACD}_{4}$ as shown by the VDG and FDS graph. This shows that the VDG and FDS graph provides a clearer picture of the performances of the prediction variance capabilities of the AOUCDs and $\mathrm{OACD}_{4}$ in the cuboidal region and should often be consider than the single-value criteria.

\section{Acknowledgements}

This work was supported by the German Academic Exchange Service (DAAD), Bonn, Germany (Funding number: 57423580 ()-0002).

\section{REFERENCES}

[1] Box G. E. P., Wilson K. B., “On the experimental attainment of optimum conditions”, Journal of the Royal Statistical Society, vol. 13, no. 1, pp. 1-45, 1951. DOI: 10.1016/0169-2070(93)90088-5

[2] Myers R. H., Montgomery D. C., Anderson-Cook C. M., "Response surface methodology: process and product optimization using designed experiments”, John Wiley and Sons, 2016.

[3] Arshad H. M., Akhtar M., Gilmour S. G., “Augmented Box-Behnken designs for fitting third-order response surfaces", Communications in Statistics-Theory and Methods, vol. 41, no. 23, pp. 4225-4239, 2012. DOI: 10.1080/03610926.2011.568154

[4] Zhang X. R., Qi Z. F., Zhou Y. D., Yang F., "Orthogonal-array composite design for the third-order models", Communications in Statistics-Theory and Methods, vol. 47, no. 14, pp. 3488-3507, 2017. DOI: 10.1080/03610926.2017.1359297

[5] Myers R. H., Vining G. G., Giovannitti-Jensen A., Myers S. L., "Variance dispersion properties of second-order response surface designs”. Journal of Quality Technology, vol. 24, no. 1, pp. 1-11, 1992. DOI: 10.1080/00224065.1992.11979368 
[6] Borkowski J. J., "Spherical prediction-variance properties of central composite and Box-Behnken designs", Technometrics, vol. 37, no. 4, pp. 399-410, 1995. DOI: 10.1080/00401706.1995.10484373

[7] Wesley W. R., Simpson J. R., Parker P. A., Pignatiello Jr J. J., "Exact calculation of integrated prediction variance for response surface designs on cuboidal and spherical regions", Journal of Quality Technology, vol. 41, no. 2, pp. 165-180, 2009a. DOI: 10.1080/00224065.2009.11917771

[8] Park Y. J., Richardson D. E., Montgomery D. C., Ozol-Godfrey A., Borror C. M., Anderson-Cook C. M., "Prediction variance properties of second-order designs for cuboidal regions”, Journal of Quality Technology, vol. 37, no. 4, pp. 253-266, 2005. DOI: 10.1080/00224065.2005.119803 29

[9] Wesley W. R., Simpson J. R., Parker P. A., Pignatiello Jr J. J. "Maximum and Minimum Prediction Variance for Spherical and Cuboidal Split Plot Designs", Communications in Statistics-Theory and Methods, vol. 38, no. 13, pp. 2251-2266, 2009b. DOI: 10.1080/03610920802521232

[10] Zahran A., Anderson-Cook C. M., Myers R. H., "Fraction of design space to assess prediction capability of response surface designs”, Journal of Quality Technology, vol. 35, no. 4, pp. 377-386, 2003. DOI: 10.1080/00224065.2003.119802 35

[11] Goldfarb H. B., Anderson-Cook C. M., Borror C. M., Montgomery D. C., "Fraction of design space plots for assessing mixture and mixture-process designs”, Journal of Quality Technology, vol. 36, no. 2, pp. 169-179, 2004. DOI: 10.1080/00224065.2004.11980263

[12] Sang - Hyun P., Dac - Heung J., "A graphical method for evaluating the effect of blocking in response surface designs", Communications in Statistics-Simulation and Computation, vol. 28, no. 2, pp. 369-380, 1999. DOI:

\section{$10.1080 / 03610919908813554$}

[13] Yankam M. B., “Augmented orthogonal uniform composite designs for the third-order models", Doctorate Thesis, University of Nigeria, Nsukka, Nigeria, 2021.

[14] Montgomery D. C., "Design and analysis of experiments", 6th Ed. Hoboken, NJ: John Wiley and Sons, Inc, 2005.

[15] Anderson-Cook C. M., Borror C. M., Montgomery D. C., "Response surface design evaluation and comparison", Journal of Statistical Planning and Inference, vol. 139, no. 2, pp. 629-41, 2009. DOI: 10.1016/j.jspi.2008.04.004

[16] Borkowski J. J., “A comparison of prediction variance criteria for response surface designs", Journal of Quality Technology, vol. 35, no. 1, pp. 70-77, 2003. DOI:10.1080/00224065.2003.11980192

[17] Giovannitti-Jensen A., Myers R. H., "Graphical assessment of the prediction capability of response surface designs", Technometrics, vol. 31, no. 2, pp. 159-171, 1989. DOI: 10.1080/00401706.1989.10488510

[18] Schoonees P., Le Roux N., Coetzer R., "Flexible graphical assessment of experimental designs in R: The vdg package", Journal of Statistical Software, vol. 74, no. 3, pp. 1-22, 2016. DOI: $10.18637 /$ jss.v074.i03

[19] Zhang A., Li H., Quan S., Yang Z., "UniDOE: Uniform Design of Experiments. R package version 1.0.2”, vol. 1, no. 2, pp. 1-12, 2018.

[20] Rady E. A., Abd El-Monsef M. M. E., Seyam M. M., "Relationship among several optimality criteria”, 2009. interstat.statjournals.net> YEAR > articles.

[21] Iwundu M. P., "Missing observations: The loss in relative A-, D-and G-efficiency”, International Journal of Advanced Mathematical Sciences, vol. 5, no. 2, pp. 43-49, 2017. DOI: 10.14419/ijams.v5i2.7786 